\title{
Erratum
}

\section{Geodesics on nonstatic Lorentz manifolds of Reissner-Nordstrom type}

\section{Fabio Giannoni}

Math. Ann. 291, 383-401 (1991)

Istituto Matematiche Applicate "U. Dini", Università di Pisa, Via Bonanno 25/B, I-56100 Pisa, Italy

Unfortunately there are various errors in this paper. Please note the corrections listed below:

Page 385, formula (1.14) should read:

$$
1 / K \geqq\left|g(z)\left[\nabla_{L} \phi(z), \nabla_{L} \phi(z)\right]\right| \geqq K,
$$

Page 385, formula (1.16) should read:

$$
\begin{gathered}
\inf \left\{\alpha_{1}(t)-\alpha_{1}^{\prime}(t)(t-\bar{t}): t, \bar{t} \in\right] a, b[\}>0, \lim _{t \rightarrow a} \alpha_{2}(t) \neq 0, \lim _{t \rightarrow b} \alpha_{2}(t) \neq 0, \\
\alpha_{3}^{\prime}(t) \leqq 0 \text { nearby } a, \quad \alpha_{3}^{\prime}(t) \geqq 0 \text { nearby } b
\end{gathered}
$$

Page 395, formula (3.1):

$$
\text { for } \leqq R\} \text { substitute } \ldots \geqq R\}
$$

Page 395, formula (3.2): should read:

$$
V_{k}=\Omega^{1} \times W_{k}, \quad F_{k}=\Omega^{1} \times B_{k},
$$

Page 395, formula (3.3):

$$
\text { for }\left(B_{k}, \partial B_{k}\right) \text { substitute }\left(W_{k}, B_{k}\right) \text {, }
$$

Page 395, remark (3.4), lines 10, 9, 8 up:

$$
\text { for } B_{k} \text { substitute } \overline{W_{k} \backslash B_{k}} \text {; }
$$

lines 7 and 5 up:

$$
\text { for }\left(B_{k}, \partial B_{k}\right) \text { substitute }\left(W_{k}, B_{k}\right) \text {, }
$$

Page 396, formula (3.10):

Page 397, formula (3.11):

$$
\text { for } \partial B_{\infty} \text { substitute } B_{\infty} \text {. }
$$

$$
\text { for } \leq R\} \text { substitute } \ldots \geq R\}
$$

Page 397: the proof of Proposition (3.13) is trivial. 